\title{
Circulating exosomal miR-125a-5p as a novel biomarker for cervical cancer
}

\author{
AIXIA LV ${ }^{1 *}, Z_{E N G R O N G ~ T U}{ }^{*}$, YUNHUA HUANG ${ }^{3}$, WEIYING LU ${ }^{3,4}$ and BAOGUO XIE \\ ${ }^{1}$ Reproductive Center, The 940th Hospital of Joint Logistics Support Force of Chinese People's Liberation Army, \\ Lanzhou, Gansu 730050; ${ }^{2}$ Reproductive Center, The First Hospital of Shanxi Medical University, Taiyuan, Shanxi 030001; \\ ${ }^{3}$ Reproductive Medicine Center, Hainan Maternal and Children's Medical Center, Haikou, Hainan 570206; ${ }^{4}$ Reproductive \\ Medicine Center, The First Affiliated Hospital of Hainan Medical University, Haikou, Hainan 570102, P.R. China
}

Received July 2, 2019; Accepted August 24, 2020

DOI: $10.3892 / \mathrm{ol} .2020 .12316$

\begin{abstract}
Exosomal microRNAs (miRs/miRNAs) have been reported to be associated with cervical cancer. The aim of the present study was to investigate circulating exosomal miRNA as a biomarker for cervical cancer diagnosis. In the present study, samples from 6 patients with cervical cancer and 6 healthy control subjects were retrieved for exosomal RNA-sequencing. The results revealed that a total of 39 miRNAs were differentially expressed between patients with cervical cancer and healthy controls $(\mathrm{P}<0.001$; fold-change $>2.0$ ). Exosomal miR-125a-5p was further quantified in plasma from 60 subjects, which included 22 healthy individuals and 38 patients with cervical cancer. miR-16a-5p served as the reference miRNA for quantitative PCR analysis of exosomal miR-125a-5p in patients with cervical cancer and healthy individuals. The results revealed that exosomal miR-125a-5p expression levels in the patients with cervical cancer were significantly lower than those in the healthy controls $(\mathrm{P}<0.001)$. Receiver operating characteristic $(\mathrm{ROC})$ curve analyses were performed and the results revealed that the level of plasma exosomal miR-125a-5p was a potential marker for differentiating between non-cervical cancer and cervical cancer, with an ROC area under the curve of 0.7129. At the cut-off value of 2.537 for miR-125a-5p, cervical cancer diagnostic sensitivities and specificities were 59.1 and $84.2 \%$, respectively. The present study provides confirmation that exosomal miR-125a-5p could potentially serve as a biomarker
\end{abstract}

Correspondence to: Dr Baoguo Xie or Dr Weiying Lu, Reproductive Medicine Center, The First Affiliated Hospital of Hainan Medical University, 31 Longhua Road, Haikou, Hainan 570102, P.R. China

E-mail: bgx1980@163.com

E-mail: 2490206511@qq.com

${ }^{*}$ Contributed equally

Key words: exosomes, microRNA-125a-5p, cervical cancer, diagnostic marker for cervical cancer diagnosis. The present study involved only a small number of clinical samples; more samples are required to support the conclusions of the present study.

\section{Introduction}

It is estimated that there were $\sim 570,000$ cases of cervical cancer and 311,000 deaths worldwide in 2018, making it the fourth most common cancer and the fourth leading cause of female cancer-associated mortality (1). There are no symptoms typically seen in the early stages of disease; therefore, novel biomarkers with high sensitivity and specificity for early detection and diagnosis are urgently required to ensure patients can receive timely treatment. Liquid biopsies, which include circulating tumor (ct)DNA, circulating tumor cells (CTCs), platelets and exosomes, are promising for early cancer detection and real time monitoring of cancer progression, response to treatment and cancer metastasis (2). Compared with CTCs and ctDNA, exosomes are advantageous in terms of stability, abundance and accessibility; exosomes are abundant in plasma and are broadly distributed in body fluids, and can be easily acquired (3). Exosomes are nano-sized and membrane-enclosed extracellular vesicles with a diameter of 30-150 nm (4). Almost all mammalian cells, including dendritic cells, adipocytes, endothelial and epithelial cells, can secrete exosomes under normal or pathological conditions (5). Exosomes carry numerous types of biological molecules, including proteins, RNAs, DNAs and lipids. Previous studies have indicated that miRNA is stably present in the exosomes, suggesting that exosomal miRNAs could be investigated as biomarkers for early diagnosis, prognosis and prediction of treatment in cancer (6). In patients with cervical cancer, homosapiens (hsa)-microRNA (miR/miRNA)-21 and hsa-miR-164a have been reported to be upregulated and associated with the high levels of cervical cancer-derived exosomes (7). It has also been reported that long non-coding RNAs HOTAIR, MALAT1 and MEG3 in exosomes can be isolated from cervicovaginal lavage and are differentially expressed in patients with cervical cancer and control patients (8).

miR-125a-5p was previously identified in normal tissue and exhibited a decreased expression in breast cancer (9). Additionally, upregulation of miR-125a-5p induced cancer 
cell apoptosis through p53 activation (10). In hepatocellular carcinoma, inhibiting miR-125a-5p increases MMP11 and vascular endothelial growth factor A protein expression, while restoring miR-125a-5p can inhibit cancer cell proliferation and metastasis (11). Circulating plasma exosomal miR-125a-3p is accessible as a diagnostic biomarker for early-stage colon cancer, which can also improve diagnostic power when combined with the serum serological tumor marker carcinoembryonic antigen (12). The aim of the present study was to determine whether cervical cancer exosomes contain low levels of miR-125a-5p by using a larger number of samples, and to evaluate whether exosomal miR-125a-5p can potentially serve as a biomarker for cervical cancer diagnosis.

\section{Materials and methods}

Patients and samples. A total of 72 individuals, including 44 patients with cervical cancer (age range, $42-65$ years; median age, 56 years) and 28 healthy controls (age range, 41-69 years; median age, 58 years), were recruited between July 2017 and November 2018 from the First Affiliated Hospital of Hainan University (Haikou, Hainan), the 940th Hospital of Joint Logistics Support Force of Chinese People's Liberation Army (Lanzhou, Gansu) and the First Hospital of Shanxi Medical University (Taiyuan, Shanxi). The clinical characteristics of the patients with cervical cancer were obtained through the hospital medical systems, including age, histological type, tumor size, the International Federation of Gynecology and Obstetrics (FIGO) stage (13), metastasis and HPV status. All cases of cervical cancer were confirmed by pathological diagnosis. The exclusion criteria were as follows: Other types of cancer besides cervical cancer, anemia, active infections, liver or kidney dysfunction, mental or psychological diseases, autoimmune disease and severe heart failure. Firstly, 12 samples were used for the exosomal small RNA sequencing, which included 6 patients with cervical cancer and 6 healthy control subjects. Next, the remaining 60 subjects, which included 22 healthy individuals and 38 patients with cervical cancer, were investigated using quantitative PCR (qPCR) to confirm the differential exosomal miRNAs. Whole blood $(10 \mathrm{ml})$ was collected by venipuncture into EDTA tubes (Guangzhou Improve Medical Instruments Co., Ltd.), centrifuged at $800 \mathrm{x} \mathrm{g}$ for $15 \mathrm{~min}$ at $4^{\circ} \mathrm{C}$, and the isolated plasma samples were stored at $-80^{\circ} \mathrm{C}$ until RNA isolation.

Exosomal RNA extraction and sequencing library preparation. Exosomes were isolated using the exoEasy Maxi kit (cat. no. 76064; Qiagen, Inc.) according to the manufacturer's instructions. Total RNA was extracted from plasma using the miRNeasy Serum/Plasma kit (cat. no. 77064; Qiagen, Inc.) according to the manufacturer's protocol and information on the label. Extracted exosomal RNA was used to prepare miRNA next-generation sequencing (NGS) libraries with the QIAseq miRNA Library kit (cat. no. 331505; Qiagen, Inc.). The sequencing was performed by HaploX Biotechnology Co., Ltd. on an Illumina HiSeq 2500 instrument. NGS data analysis was performed using the GeneReader system (Qiagen, Inc.) according to manufacturer's instructions (14).
Reverse transcription-qPCR. Total RNA was extracted from exosomes using a miRNeasy kit (cat. no. 217184; Qiagen, Inc.). A total of $200 \mu \mathrm{l}$ plasma was mixed with QIAzol Lysis Reagent (cat. no. 79306; Qiagen, Inc.) according to the manufacturer's instructions. Exosomal RNA was reverse transcribed using a Qiagen miRNA Reverse Transcription kit (Qiagen, Inc.) according to the manufacturer's instructions. The levels of miRNA $125-5 \mathrm{p}$ were determined in triplicate and analyzed using a Real-time PCR detection system (LineGene K Plus; Hangzhou Bioer Co., Ltd.) using SYBR green qPCRmaster mix (Qiagen, Inc.). PCR was performed in 40 cycles with each cycle consisting of $30 \mathrm{sec}$ at $94^{\circ} \mathrm{C}$ and $30 \mathrm{sec}$ at $62^{\circ} \mathrm{C}$. The PCR data were normalized using the $2^{-\Delta \Delta \mathrm{Cq}}$ value method (15), using hsa-miR-16-5p for normalization $(16,17)$. The primers used for the amplification were as follows: hsa-miR-125a-5p, forward, 5'-ACACTCCAGCTGGGTCCCTGAGACCCT TTAAC-3' and reverse, 5'-TGGTGTCGTGGAGTCG-3'; hsa-miR-16-5p, forward, 5'-TAGCAGCACGTAAATAT TGGCG-3' and reverse, 5'-TGCGTGTCGTGGAGTC-3'.

ZetaView nanoparticle tracking analysis (NTA). The present study measured exosome particle size and concentration with a ZetaView PMX v110 (Particle Metrix GmbH) using the settings recommended by the manufacturer's software manual. For each exosomal sample, $2 \mathrm{ml}$ of the sample was diluted using 1X PBS buffer, the NTA measurement was recorded and analyzed, and the mean, median and mode, as well as the concentration of the sample, were calculated using ZetaView software (version 8.02.28; Particle Metrix GmbH).

Transmission electron microscopy (TEM). For TEM, $20 \mu 1$ of the exosomal sample was fixed to an electron microscopy mesh grid with $2 \%$ glutaraldehyde at room temperature, so that the dropping liquid remained on the copper net for $20 \mathrm{~min}$. Subsequently, $2 \%$ aqueous solution of uranyl acetate was used to stain samples for $5 \mathrm{~min}$ at room temperature, and the filter paper was air-dried at room temperature and observed under a $120 \mathrm{kv}$ biotransmission electron microscope (magnification, $\mathrm{x} 60,000)$.

Statistical analysis. Statistical analysis was performed using R software (version 3.5.0; https://www.R-project.org). The miRNA experiments were performed in triplicate and the results are presented as the mean \pm standard deviation. Associations between exosomal miR-125a-5p expression and clinicopathological characteristics were analyzed using unpaired Student's t-test. The heat map was created using the pheatmap R package (version 1.0.12; https://www. rdocumentation.org/packages/pheatmap) and the receiver operating characteristic (ROC) curve analyses were plotted using the ggplot2 R package (version 3.3.2; CRAN.R-project. org/package $=$ ggplot 2 ). The cut-off values were calculated using Youden's index. $\mathrm{P}<0.05$ was considered to indicate a statistically significant difference.

\section{Results}

Characterization of isolated exosomes. The results of the present study demonstrated that exosomes had a spherical shape with a diameter of $30-200 \mathrm{~nm}$, as indicated by TEM, and the size 

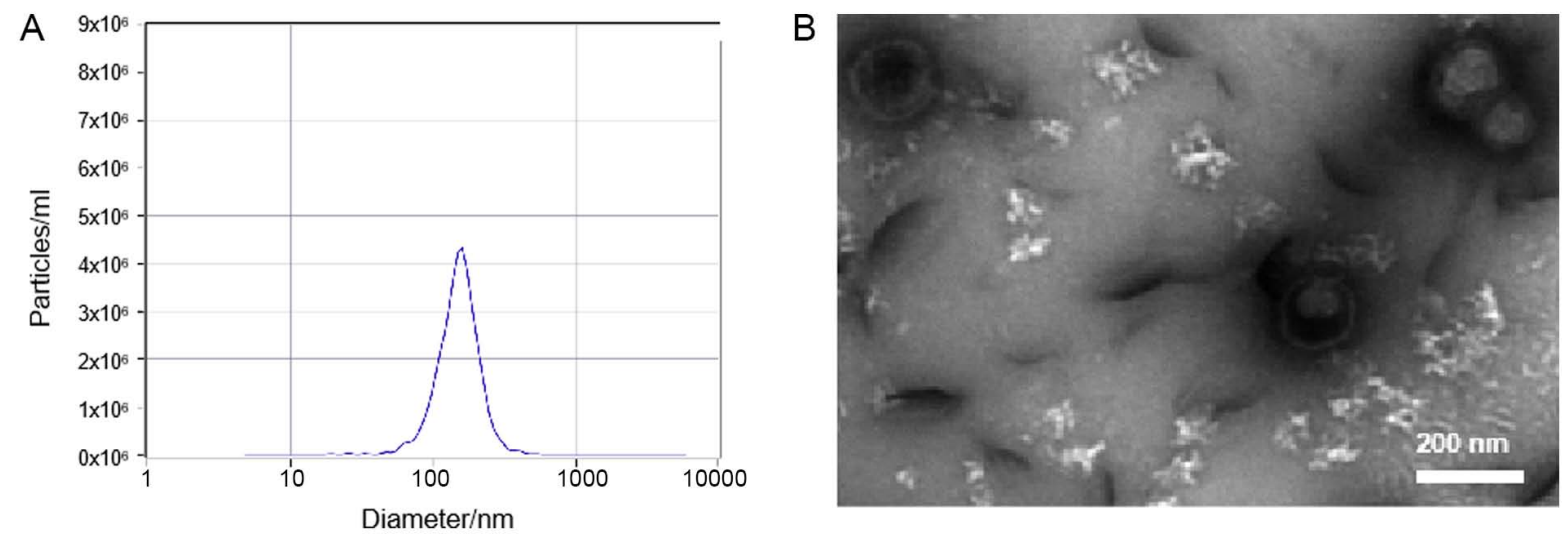

Figure 1. Identification of exosomes purified from the plasma of patients with cervical cancer. (A) Nanoparticle Tracking Analysis determined the size of exosomes. (B) Exosomes were analyzed under electron microscopy and highlighted with red arrows, with diameter sizes ranging from 50 to $180 \mathrm{~nm}$.
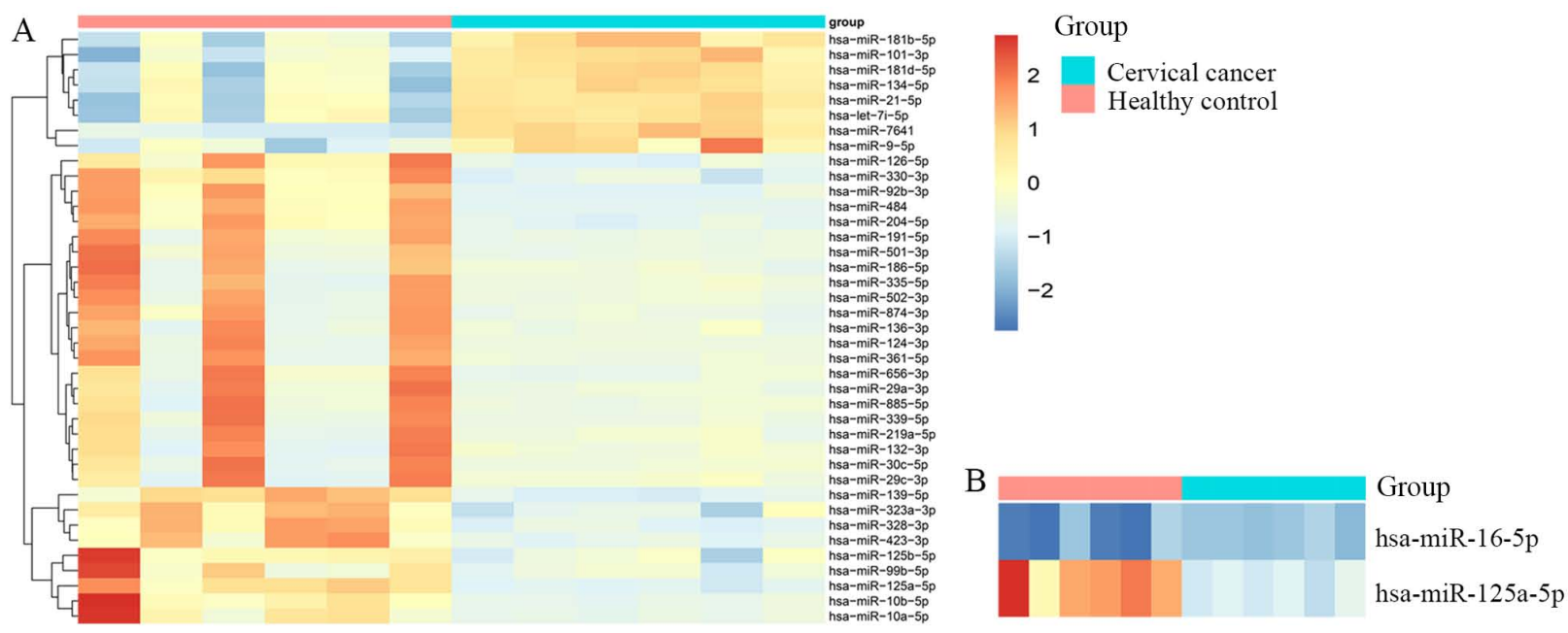

Figure 2. RNA profiling of exosome samples based on differentially expressed miRNAs. (A) Heat map presenting unsupervised hierarchical clustering of miRNAs. Red indicates miRNAs with high expression levels and blue indicates miRNAs with low expression levels. (B) The expression of hsa-miR-16-5p was stable, while the expression of has-miR-125a-5p was very different in patients with cervical cancer and healthy controls. miRNA/miR, microRNA; hsa, homosapiens.

distribution of exosomes ranged from 50 to $180 \mathrm{~nm}$ in diameter, with a mode value of $140-150 \mathrm{~nm}$, as indicated by NTA (Fig. 1).

ExosomalmiRNA profiling. The present study performed plasma exosomal miRNA profiling for 6 patients with cervical cancer and 6 matched healthy controls. A total of 1,725 unique mature miRNAs were identified from exosome miRNA sequencing data, and 167 miRNAs were included for statistical analysis after removing the miRNAs with $<100$ average reads per sample. The results of the statistical analysis revealed that 39 miRNAs were differentially expressed between patients with cervical cancer and healthy controls, among which, 8 miRNAs were downregulated and 31 miRNAs were upregulated $(\mathrm{P}<0.001$; fold-change $>2.0$; Fig. 2A). Considering these exosomal miRNA expression patterns, and evidence obtained from previous studies $(7,8)$, miR-125a-5p was selected for the further analysis of its prognostic values in cervical cancer. In the present study, miR-16a-5p had a small standard deviation, and was also used as an endogenous reference in previously published studies $(15,16)$ (Fig. 2B). Thus, miR-16a-5p was selected as an endogenous reference in the subsequent qPCR assay.
Prognostic role of plasma exosomal miR-125a-5p expression levels in patients with cervical cancer. To assess the potential prognostic role of exosomal miRNA expression levels, miR-125a-5p was quantified in the plasma of 60 subjects, which included 22 healthy individuals and 38 patients with cervical cancer. The results revealed that exosomal miR-125a-5p expression levels in patients with cervical cancer were significantly lower than those in healthy controls $(\mathrm{P}<0.001)$. ROC curve analyses were performed to evaluate the diagnostic value of exosomal miR-125a-5p for cervical cancer. These analyses revealed that the level of plasma exosomal miR-125a-5p was a potential marker for differentiating between non-cervical and cervical cancer, with an ROC area under the curve value of 0.7129 [95\% confidence interval (CI), 0.561-0.865] (Fig. 3B). At the cut-off value of 2.537 for miR-125a-5p, cervical cancer diagnostic sensitivities and specificities were 59.1 and $84.2 \%$, respectively.

Clinicopathological characteristics and miR-125a-5p profile. miR-125a-5p was significantly associated with age, tumor size and FIGO stage. Compared with HPV-negative patients, 
Table I. Association between exosomal miR-125a-5p expression levels and the clinicopathological characteristics of patients with cervical cancer.

\begin{tabular}{|c|c|c|c|c|}
\hline Characteristic & Number of patients & miR-125a-5p expression ${ }^{a}$ & T-value & P-value \\
\hline Age, years & & & 3.461 & 0.0032 \\
\hline$<50$ & 6 & $0.76 \pm 0.41$ & & \\
\hline$\geq 50$ & 32 & $1.57 \pm 0.92$ & & \\
\hline Histological type & & & 0.989 & 0.3361 \\
\hline SCC & 28 & $1.53 \pm 0.93$ & & \\
\hline SDC & 10 & $1.22 \pm 0.84$ & & \\
\hline Tumor size, $\mathrm{cm}$ & & & 3.155 & 0.0039 \\
\hline$<4$ & 29 & $0.88 \pm 0.48$ & & \\
\hline$\geq 4$ & 9 & $1.62 \pm 0.94$ & & \\
\hline FIGO stage & & & 2.475 & 0.0217 \\
\hline IA-IIA & 29 & $0.95 \pm 0.59$ & & \\
\hline IIB-IVB & 9 & $1.60 \pm 0.94$ & & \\
\hline Lymph node metastasis & & & 1.312 & 0.1978 \\
\hline Yes & 22 & $1.60 \pm 0.99$ & & \\
\hline No & 16 & $1.23 \pm 0.73$ & & \\
\hline HPV status & & & 2.111 & 0.0418 \\
\hline Positive & 30 & $1.29 \pm 0.83$ & & \\
\hline Negative & 8 & $2.02 \pm 0.88$ & & \\
\hline
\end{tabular}

${ }^{\mathrm{a}}$ Mean \pm SD. miR, microRNA; SCC, cervical squamous cell carcinoma; ADC, adenocarcinoma; FIGO, International Federation of Gynaecology and Obstetrics; HPV, human papillomavirus.
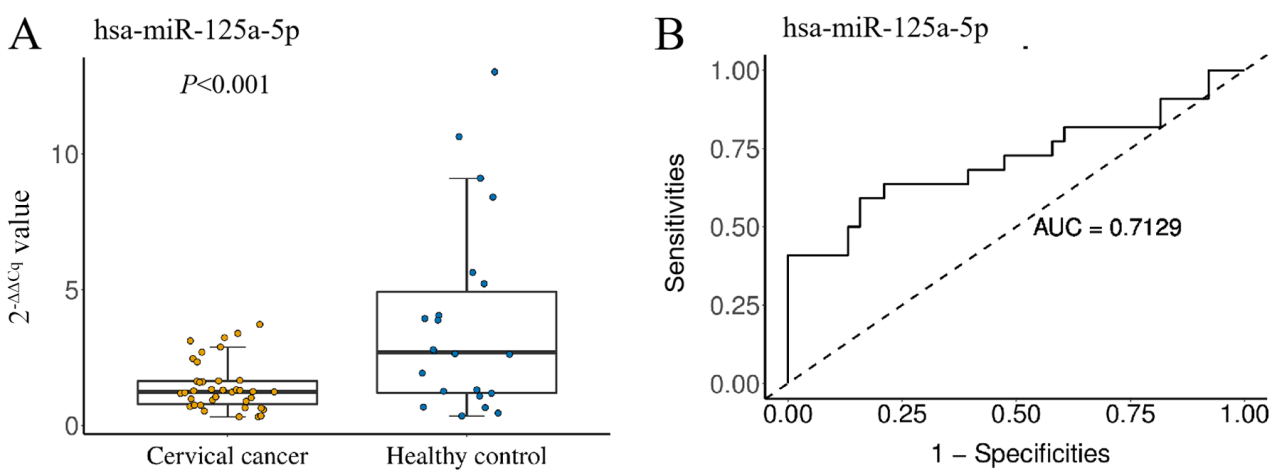

Figure 3. Receiver operating characteristic curve analysis displaying the diagnostic power of exosomal miR-125a-5p. (A) The relative plasma exosomal miR-125a-5p expression levels were compared between patients with cervical cancer $(n=38)$ and healthy controls $(n=22)$. The exosomal miR-125a-5p expression levels in patients with cervical cancer were significantly lower than those in healthy controls $(\mathrm{P}<0.001)$. (B) A receiver operator characteristic curve analysis for determining a cut-off value for differentiating between normal and cancer cells was performed. The AUC value was 0.7129 (95\% confidence interval, 0.561-0.865), with a cut-off value of 2.537. miR, microRNA; AUC, area under the curve; hsa, homosapien.

miR-125a-5p expression in HPV-positive patients was significantly decreased. However, there was no significant difference between miR-125a-5p expression and lymph node metastasis or histological types (Table I).

\section{Discussion}

Cervical cancer is a common tumor in gynecology. The early screening techniques for cervical cancer include high-risk human papillomavirus (HR-HPV) testing and cervical exfoliation cell examination (18). As certain persistent HR-HPV subtype infections can develop into cervical cancer, the HR-HPV test is highly sensitive and has low specificity in screening for cervical cancer (19). By contrast, cytology has an improved specificity for cervical cancer screening, and cervical screening strategies for women based on cytology are safe and effective (20). Exosomes are a subvesicle structure secreted by a variety of cells in the body; they carry a variety of proteins and nucleic acid substances from cells, and can be easily obtained from peripheral blood and other body fluids, such as joint cavity effusion and pleural effusion $(21,22)$. Thus, in recent years, exosomes have been used as biomarkers for disease diagnosis (23). 
It has been demonstrated that miRNAs are likely to be transported through exosomes in body fluids (24). When exosomes are released from the original cells, they fuse with the recipient cells and control the gene expression, which increases the possibility of potential tumorigenicity of exosomal miRNA $(25,26)$. In a clinical study (4), exosomal miR-1290 and miR-375 have been reported as promising prognostic biomarkers for castration-resistant prostate cancer. Liu et al (27) determined that exosomal circ_0047921, circ_0056285 and circ_0007761 were promising biomarkers for the diagnosis of non-small cell lung carcinoma, including the early stages of disease; in addition, exosomal circ_0056285 was associated with clinical stage and lymph node metastasis. However, serum exosome-derived miRNAs are different from the total serum miRNAs. A previous study determined that there were no correlations between serum miR-126 and exosomal miR-126 (28), and another study demonstrated that serum miR-122 and miR-199a were potential biomarkers that reflect antiviral therapy efficacy in patients with hepatitis C (29); however, to the best of our knowledge, there is no evidence that exosomal miR-122 and miR-199a have the same predictive effect.

A previous study has revealed that miR-125a-5p expression was decreased in clinical gastric cancer tissue samples and gastric cancer cell lines, and was associated with tumorigenesis and poor prognosis (30). Furthermore, an experimental study involving molecular mechanisms have demonstrated that miR-125a-5p inhibited the proliferation and invasion of ovarian cancer cells by targeting GALNT14 (31), and inhibited the invasion and migration of hepatoma cells by regulating the activity of the PI3K/AKT/mTOR signaling pathway (32). In addition, a previous study revealed that HPV suppresses miR-125a-5p by inhibiting p53 expression in cervical carcinogenesis, and it is speculated that miR-125a-5p may be a therapeutic target for cervical cancer (33). However, to the best of our knowledge, there are no studies on miR-125a-5p in cervical cancer.

The present study performed plasma exosomal miRNA sequencing, the results of which revealed that a total of 39 miRNAs were differentially expressed between patients with cervical cancer and healthy controls, among which, 8 miRNAs were downregulated and $31 \mathrm{miRNAs}$ were upregulated with the statistical criteria of $\mathrm{P}<0.001$, fold-change $>2.0$. Based on both NGS and PCR experiments, exosomal miR-125a-5p appears to be a promising biomarker. Diagnostic accuracy was calculated based on the PCR results $(0.7129 ; 95 \% \mathrm{CI}$, $0.561-0.865)$. The present study revealed that miR-125a-5p was significantly associated with age; 6 patients $<50$ years of age had a lower miR-125a-5p level compared with 32 patients $\geq 50$ years of age. In addition, tumor size and FIGO stage were associated with miR-125a-5p level, but no differences were observed between cervical squamous cell carcinoma and adenocarcinoma/adenosquamous carcinoma. However, it should be noted that exosomal miRNAs do not necessarily represent cellular levels, since the miRNA sorting mechanism may affect the incorporation of miRNA into exosomes. It should also be noted that the present study contained a small number of samples and more samples are required to support the conclusions of the present study.

As previously reported, miR-125a-5p could be a potential biomarker of certain types of cancer, such as breast (9), hepatocellular (34) and colon cancer (12), which indicates that miR-125a-5p is not a cervical cancer specific biomarker. The present study suggested that when miR-125a-5p expression is decreased, miR-125a-5p cannot be relied on to determine which type of cancer, but it may provide auxiliary diagnostic value for clinical samples with an uncertain diagnosis. Liquid biopsy is a new area of research, and it is therefore not appropriate for them to replace traditional methods in diagnosing cancer, including exosome-based diagnosis and screening programs. However, it is still of great scientific value to investigate novel approaches to cancer diagnosis, including exosomal miRNAs. However, the present study has several limitations. Different histological types of cervical cancer may have different molecular mechanisms and different circulating exosomal miRNAs. Future studies should focus on one histological subtype, squamous cell carcinoma or adenocarcinoma, and include more samples to further confirm the present results.

In conclusion, the results of the present study revealed that plasma exosomal miR-125a-5p is poorly expressed in cervical cancer and that miR-125a-5p could be regarded as a diagnostic marker for cervical cancer. Further research with larger sample sizes is required to confirm these results.

\section{Acknowledgements}

Not applicable.

\section{Funding}

The present study was funded by the Major Science and Technology Program of Hainan Province (grant no. ZDKJ2017007), the Natural Science Foundation of China (grant no. 81560244), and the Key Research and Development Program of Hainan Province (grant no. ZDYF2019158)

\section{Availability of data and materials}

The datasets used and/or analyzed during the current study are available from the corresponding author on reasonable request.

\section{Authors' contributions}

BX and YH designed the study. AL and ZT collected the clinical data and participated the writing work. YH, AL and WL were responsible for the RNA-sequencing data and PCR processing and analysis. All authors read and approved the final manuscript.

\section{Ethics approval and consent to participate}

The Ethics Committee of the First Affiliated Hospital of Hainan University approved the present study. All samples were anonymized during the analysis. Written informed consent of blinded individuals was obtained from each participant.

\section{Patient consent for publication}

Not applicable. 


\section{Competing interests}

The authors declare that they have no competing interests.

\section{References}

1. Bray F, Ferlay J, Soerjomataram I, Siegel RL, Torre LA and Jemal A: Global cancer statistics 2018: GLOBOCAN estimates of incidence and mortality worldwide for 36 cancers in 185 countries. CA Cancer J Clin 68: 394-424, 2018.

2. De Rubis G, Rajeev Krishnan S and Bebawy M: Liquid biopsies in cancer diagnosis, monitoring, and prognosis. Trends Pharmacol Sci 40: 172-186, 2019

3. Baassiri A, Nassar F, Mukherji D, Shamseddine A, Nasr R and Temraz S: Exosomal Non Coding RNA in LIQUID Biopsies as a Promising Biomarker for Colorectal Cancer. Int J Mol Sci 21: 1398,2020

4. Huang X, Yuan T, Liang M, Du M, Xia S, Dittmar R, Wang D, See W, Costello BA, Quevedo F, et al: Exosomal miR-1290 and miR-375 as prognostic markers in castration-resistant prostate cancer. Eur Urol 67: 33-41, 2015.

5. Camussi G, Deregibus MC, Bruno S, Cantaluppi V and Biancone L: Exosomes/microvesicles as a mechanism of cell-to-cell communication. Kidney Int 78: 838-848, 2010.

6. Jiang L, Gu Y, Du Y and Liu J: Exosomes: Diagnostic biomarkers and therapeutic delivery vehicles for cancer. Mol Pharm 16: $3333-3349,2019$

7. Liu J, Sun H, Wang X, Yu Q, Li S, Yu X and Gong W: Increased exosomal microRNA-21 and microRNA-146a levels in the cervicovaginal lavage specimens of patients with cervical cancer. Int J Mol Sci 15: 758-773, 2014.

8. Zhang J, Liu SC, Luo XH, Tao GX, Guan M, Yuan H and Hu DK: Exosomal long noncoding RNAs are differentially expressed in the cervicovaginal lavage samples of cervical cancer patients. J Clin Lab Anal 30: 1116-1121, 2016.

9. Iorio MV, Ferracin M, Liu CG, Veronese A, Spizzo R, Sabbioni S, Magri E, Pedriali M, Fabbri M, Campiglio M, et al: MicroRNA gene expression deregulation in human breast cancer. Cancer Res 65: 7065-7070, 2005.

10. Jiang L, Huang Q, Chang J, Wang E and Qiu X: MicroRNA HSA-miR-125a-5p induces apoptosis by activating p53 in lung cancer cells. Exp Lung Res 37: 387-398, 2011.

11. Bi Q, Tang S, Xia L, Du R, Fan R, Gao L, Jin J, Liang S, Chen Z, $\mathrm{Xu}$ G, et al: Ectopic expression of MiR-125a inhibits the proliferation and metastasis of hepatocellular carcinoma by targeting MMP11 and VEGF. PLoS One 7: e40169, 2012.

12. Wang J, Yan F, Zhao Q, Zhan F, Wang R, Wang L, Zhang Y and Huang X: Circulating exosomal miR-125a-3p as a novel biomarker for early-stage colon cancer. Sci Rep 7: 4150, 2017.

13. Matsuo K, Machida H, Mandelbaum RS, Konishi I and Mikami M: Validation of the 2018 FIGO cervical cancer staging system. Gynecol Oncol 152: 87-93, 2019.

14. Koitzsch U,Heydt C, Attig H,Immerschitt I, Merkelbach-Bruse S, Fammartino A, Büttner RH, Kong Y and Odenthal M: Use of the GeneReader NGS System in a clinical pathology laboratory: A comparative study. J Clin Pathol 70: 725-728, 2017.

15. Livak KJ and Schmittgen TD: Analysis of relative gene expression data using real-time quantitative PCR and the 2(- $\Delta \Delta$ C(T)) Method. Methods 25: 402-408, 2001.

16. Shahid S, Shaheen J, Shahid W, Akhtar MW and Sadaf S: mir-16-5p as a suitable reference gene for normalization of quantitative real time PCR in acute lymphoblastic leukemia. Pak J Zool 51: 747-754, 2019

17. Lange T, Stracke S, Rettig R, Lendeckel U, Kuhn J, Schlüter R, Rippe V, Endlich K and Endlich N: Identification of miR-16 as an endogenous reference gene for the normalization of urinary exosomal miRNA expression data from CKD patients. PLoS One 12: e0183435, 2017.

18. De Vuyst H, Chung MH, Baussano I, Mugo NR, Tenet V, van Kemenade FJ, Rana FS, Sakr SR, Meijer CJLM, Snijders PJF, et al: Comparison of HPV DNA testing in cervical exfoliated cells and tissue biopsies among HIV-positive women in Kenya. Int J Cancer 133: 1441-1446, 2013.
19. Benevolo M, Vocaturo A, Caraceni D, French D, Rosini S, Zappacosta R, Terrenato I, Ciccocioppo L, Frega A and Giorgi Rossi P: Sensitivity, specificity, and clinical value of human papillomavirus (HPV) E6/E7 mRNA assay as a triage test for cervical cytology and HPV DNA test. J Clin Microbiol 49: 2643-2650, 2011.

20. Dillner J, Rebolj M, Birembaut P, Petry KU, Szarewski A, Munk C, de Sanjose S, Naucler P, Lloveras B, Kjaer S, et al; Joint European Cohort Study: Long term predictive values of cytology and human papillomavirus testing in cervical cancer screening: Joint European cohort study. BMJ 337 (oct13 1): a1754, 2008.

21. Bard MP, Hegmans JP, Hemmes A, Luider TM, Willemsen R, Severijnen LAA, van Meerbeeck JP, Burgers SA, Hoogsteden HC and Lambrecht $\mathrm{BN}$ : Proteomic analysis of exosomes isolated from human malignant pleural effusions. Am J Respir Cell Mol Biol 31: 114-121, 2004.

22. Keller S, Ridinger J, Rupp AK, Janssen JW and Altevogt P: Body fluid derived exosomes as a novel template for clinical diagnostics. J Transl Med 9: 86, 2011.

23. Jalalian SH, Ramezani M, Jalalian SA, Abnous $\mathrm{K}$ and Taghdisi SM: Exosomes, new biomarkers in early cancer detection. Anal Biochem 571: 1-13, 2019.

24. Salido-Guadarrama I, Romero-Cordoba S, Peralta-Zaragoza O, Hidalgo-Miranda A and Rodríguez-Dorantes M: MicroRNAs transported by exosomes in body fluids as mediators of intercellular communication in cancer. Onco Targets Ther 7: 1327-1338, 2014.

25. Ahmadi M and Rezaie J: Tumor cells derived-exosomes as angiogenenic agents: Possible therapeutic implications. J Transl Med 18: 249, 2020

26. Ghaemmaghami AB, Mahjoubin-Tehran M, Movahedpour A, Morshedi K, Sheida A, Taghavi SP, Mirzaei H and Hamblin MR: Role of exosomes in malignant glioma: microRNAs and proteins in pathogenesis and diagnosis. Cell Commun Signal 18: 120, 2020.

27. Liu L, Su W, Xian J, Wang Y, Rao B, Lin M and Chen J: Identification of Three Circular RNAs Cargo in Serum Exosomes as Diagnostic Biomarkers of Non-Small Cell. Lung Cancer, 2018.

28. Chen F, Du Y, Esposito E, Liu Y, Guo S, Wang X, Lo EH, Xing C and Ji X: Effects of focal cerebral ischemia on exosomal versus serum miR126. Transl Stroke Res 6: 478-484, 2015.

29. Jiao X, Fan Z, Chen H, He P, Li Y, Zhang Q and Ke C: Serum and exosomal miR-122 and miR-199a as a biomarker to predict therapeutic efficacy of hepatitis C patients. J Med Virol 89: 1597-1605, 2017.

30. Cao Y, Tan S, Tu Y, Zhang G, Liu Y, Li D, Xu S, Le Z, Xiong J, Zou W, et al: MicroRNA-125a-5p inhibits invasion and metastasis of gastric cancer cells by targeting BRMS1 expression. Oncol Lett 15: 5119-5130, 2018.

31. Yang J, Li G and Zhang K: MiR-125a regulates ovarian cancer proliferation and invasion by repressing GALNT14 expression. Biomed Pharmacother 80: 381-387, 2016.

32. Kang S, Dong SM, Kim BR, Park MS, Trink B, Byun HJ and Rho SB: Thioridazine induces apoptosis by targeting the $\mathrm{PI} 3 \mathrm{~K} / \mathrm{Akt} / \mathrm{mTOR}$ pathway in cervical and endometrial cancer cells. Apoptosis 17: 989-997, 2012.

33. Fan Z, Cui H, Xu X, Lin Z, Zhang X, Kang L, Han B, Meng J, Yan Z, Yan X, et al: MiR-125a suppresses tumor growth, invasion and metastasis in cervical cancer by targeting STAT3. Oncotarget 6: 25266-25280, 2015.

34. Kim JK, Noh JH, Jung KH, Eun JW, Bae HJ, Kim MG, Chang YG, Shen Q, Park WS, Lee JY, et al: Sirtuin7 oncogenic potential in human hepatocellular carcinoma and its regulation by the tumor suppressors MiR-125a-5p and MiR-125b. Hepatology 57: $1055-1067,2013$

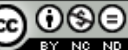

This work is licensed under a Creative Commons Attribution-NonCommercial-NoDerivatives 4.0 International (CC BY-NC-ND 4.0) License. 